not recorded to date. These expected species would include Roadside Skipper, Peck's Skipper, Long Dash, Arctic Skipper, Northern Cloudy Wing, Giant Sulphur, Sara Orange Tip, Zephyr Angle Wing and Variegated Fritillary.

\section{Acknowledgements}

I would like to thank Norbert Kondla for supplying me with his collection data, and for reviewing the manuscript.

\footnotetext{
BIRD, C. D. 1975. A calendar of the butterflies and skippers of the alpine area of Plateau Mountain. Alberta Naturalist 5:26-28.

${ }^{2}$ BIRD, C. D. 1975. A calendar of the skippers and butterflies of Banff National Park. Alberta Naturalist 5:7175.
}

${ }^{3}$ BIRD, C. D. 1975. A revised calendar of the butterflies and skippers of Calgary. Calgary Field Naturalist 6:312-314.

${ }^{4}$ HOOPER, R. R. 1973. The butterflies of Saskatchewan. Saskatchewan Museum of Natural History. 216 pp.

${ }^{5}$ HOWE, W. H. 1975. The Butterflies of North America. Doubleday \& Company Inc., New York, 633 pp.

${ }^{6}$ KONDLA, N. G. and C. D. BIRD. 1979. The skippers and butterflies of Kananaskis Provincial Park, Alberta. Blue Jay 37:73-85.

${ }^{7}$ MILLER, L. O. and F. M. BROWN. 1981. A Catalogue/Checklist of the Butterflies of America North of Mexico. The Lepidopterists Society, Memoir No. 2. $280 \mathrm{pp}$.

\title{
LACEWINGS AND APHIS LIONS
}

\section{G. PUTNAM, 35 Kirk Crescent, Saskatoon, Saskatchewan. S7H 3B1}

One of the author's principal research concerns was the diamondback moth and its larvae (Plutella xylostella L. (Plutellidae)), an occasional pest of Brassica seed crops in Saskatchewan. In an earlier paper in this journal, I briefly described two hymenopterous parasites of diamondback larvae. Here, I wish to write about another naturally occurring biological control agent, one predator that seemed potentially significant in the natural control of the diamondback. It is one of the lacewings, Chrysopa carnea (Neuroptera: Chrysopidae).

Lacewing larvae are commonly called aphis lions, aphids being a common prey. Aphis lions are active little creatures, their appearance suggesting tiny alligators, with their mouth parts extended to form a pair of "pincers", with which they puncture and drain the juices out of anything defenceless and tender enough to handle. They will sometimes even take a pinch at one's bare arm, no doubt trying it for flavour and tenderness. Completing larval growth rather quickly under good conditions, they spin a small spherical cocoon resembling a miniature tennis ball. Out of this soon emerges a pale green adult lacewing, seeming to be much too bulky ever to have developed in such a tiny space. They are occasionally seen in open spaces in low, clumsy-looking flight.

It was known that aphis lions were broad spectrum feeders, but small scale experiments were first set up for assurance that they would feed on diamondback eggs (easy targets), larvae alone, and especially, larvae in the presence of aphids. It was thought that aphids might be a preferred food, but it turned out that the predators did not discriminate.

The next step was to set up a cage 
experiment in biological control, in the greenhouse. This was done with some background experience with rearing both prey and predator; the former was extremely easy to rear in a confined space, the latter almost as easy, if some debris was provided as concealment from cannibalism, which was probably never completely avoided. Potted winter rapeseed plants were used as food for the diamondbacks, as they remained vegetative. On Day 1, one male and one female moth, newly emerged, were entered in the experiment; this was repeated on Day 7 . In the period Day 3 21,14 entries of young chrysopid larvae were entered at the rate of five per entry. By Day 20, all surviving diamondback progeny, a total of seven, had emerged as moths. There are no data on how many eggs and larvae were predated. By Day 35, 18 lacewing adults had emerged, a yield of $26 \%$. On the same day, the next generation of diamondbacks was entering pupation.

About 165 3rd generation moths emerged. From these, uncounted thousands of blotch leaf mines, originally containing the hatchling diamondback larvae, were visible on the food plant foliage. The new crop of aphis lions, synchronous with the prey larvae, cleaned up virtually all of them, along with any aphids present. The result was quite dramatic.

In retrospect, the experiment described above turned out to have been programmed, as it were, to produce the observed result. Both predator and prey were in unnaturally high concentrations, to mention only one unnatural feature of the experiment. The main point about it was that while the diamondback (prey) component in the culture was into its third generation, the lacewing (predator) part was into its second. Thus the second generation prey larvae were missed altogether. If this differential developmental rate applies to the slower developing field conditions as well, the second larval generation of diamondbacks, which is the crucial one from the point of view of potential damage, would be unaffected. There may be some significance to this, as aphis lions have been taken in sweep netting at a time too late to be useful as agents against diamondbacks. However this is an observation that needs much more thorough scrutiny. Aphis lions develop quickly in the presence of ample food, and the pupal stage in the cocoon does not seem long, but, as some authors have claimed, the pre-oviposition period in the adult seems lengthy. ${ }^{1}$

C. carnea, as an insect-eating predator, has received a lot of research attention in recent decades. Entomologists in California developed a mass rearing technique based on feeding the aphis lions on eggs and larvae of the potato tuber moth, and the adults on foods formulated to improve egg production. A few years ago, and perhaps still, C. carnea material could be purchased commercially for mass release. One application was for the control of mealy bugs on high value fruit trees. Experimental releases on field plots for the control of certain cotton insects were reported to be successful. It is difficult to see how such a release procedure could be applied on a field scale against, for example, diamondback larvae in a million-hectare crop. Meanwhile, although the cost-benefit ratio of such an investigation might be questionable, it would be interesting to examine the bionomics of this predator in our rapeseed/canola fields with care.

The author carried out the experiments described above while in the employ of the Agriculture Canada Research Station, 107 Science Crescent, Saskatoon, Saskatchewan S7N 0X2, using the Station's facilities.

' TAUBER, M. J., CATHERINE A. TAUBER, and CHARLENE J. DENYS. 1970. Adult diapause in Chrysopa carnea: Photoperiodic control of duration and colour. J. Insect Phys. 16:949-955. 